\title{
Exploring the Kibble-Zurek mechanism in a secondary bifurcation
}

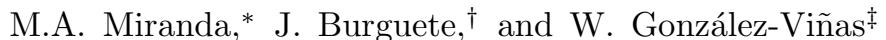 \\ Dept. of Physics and Applied Mathematics, Universidad de Navarra. Irunlarrea s/n, E-31080 Pamplona, Spain \\ H. Mancinß \\ Institute of Physics, University of Navarra. Irunlarrea s/n, E-31080 Pamplona, Spain
}

\begin{abstract}
We present new experimental results on the quenching dynamics of an extended thermo-convective system (a network array of approximately 100 convective oscillators) going through a secondary subcritical bifurcation. We characterize a dynamical phase transition through the nature of the domain walls (1D-fronts) that connect the basic multicellular pattern with the new oscillating one. Two different mechanisms of the relaxing dynamics at the threshold are characterized depending on the crossing rate $\mu=\left.\frac{d \varepsilon}{d t}\right|_{\varepsilon=0}$ (where $\varepsilon$ is the control parameter) of the quenched transition. From the analysis of fronts, we show that these mechanisms follow different correlation length scales $\xi \sim \mu^{-\sigma}$. Below a critical value $\mu_{c}$ a slow response dynamics yields a spatiotemporal coherent front with weak coupling between oscillators. Above $\mu_{c}$, for rapid quenches, defects are trapped at the front with a strong coupling between oscillators, similarly to the Kibble-Zurek mechanism in quenched phase transitions. These defects, which are pinned to the fronts, yield a strong decay of the correlation length.
\end{abstract}

Keywords: Synchronization, pattern formation, non-equilibrium phase transitions, Networks, front dynamics, symmetry breaking bifurcations, cosmology.

Note: A version of this article has been accepted for publication in the Int. J. Bifurcation and Chaos

\section{INTRODUCTION}

A dynamical synchronization transition is the closest expression of a 'real' phase transition in nature. Some recent interest on synchronization processes [1-10] is meant to understand the interaction between oscillating units which give rise to a collective behavior (i.e. as in a phase transition or in a Network). A key aspect of most phase transitions is the breaking of symmetries which should be inherited in the synchronization processes.

Moreover, in nature, systems that undertake symmetry breaking transitions show a richer phenomenology. As a matter of fact, it has been argued that in the early universe, short after the Big Bang this kind of transitions had made possible the appearance of the present electromagnetic field from unified fields, and the asymmetry between matter and antimatter in the universe [1113], among other important phenomena. Kibble [12] proposed that cosmological phase transitions which underwent in the early universe could also be responsible for the large scale structure that is observed in the present time (i.e. distribution of galaxies). This may happen due to the existence of many causally uncorrelated regions in a less symmetric phase after the transitions. This will lead to a phase mismatch and thus, to the appearance of

\footnotetext{
*e-mail address: montse@alumni.unav.es

†e-mail address: javier@fisica.unav.es

‡e-mail address: wens@fisica.unav.es

$\S$ e-mail address: hmancini@fisica.unav.es
}

phase singularities (topological defects). Those defects determined huge energy fluctuations which could be related to the large scale of the universe, and even to the existence of topological dark matter [14].

Later, Zurek [15, 16] proved that this causal mechanism has its counterpart in condensed matter systems, and in general can be applied to all second order breaking phase transitions. The generalized mechanism is known as the Kibble-Zurek one. The argument is the following: on one hand, when the control parameter is far from the critical point, any slow change in the control parameter would be followed adiabatically by the states of the system. On the other hand, in the critical region the relaxation time diverges. Thus, the system cannot follow the control parameter because otherwise the fluctuations should propagate faster than the limiting speed in the system. Consequently the correlation length of the fluctuations gets frozen until the adiabatic dynamics is restored (after the transition is crossed). Then, fluctuations grow to form the new phase, and topological defects appear due to the aforementioned mismatch. This kind of defects, due to their topological stability, keep the correlation values until well after the transition is performed. This mechanism leads to a power law for the correlation length as a function of the rate of change of the control parameter.

Many condensed matter experiments have been performed to confirm cosmological theories in the laboratory [15, 17 19]. Among them, in liquid crystals [20 22], in superfluid helium [23 30], in superconductors and Josephson junctions 31 38], in Bose-Einstein condensates [39, 40] and in other condensed matter systems [41]. 
Numerical and theoretical approaches have been numerous.

The Kibble-Zurek mechanism has also been extended to non-equilibrium primary bifurcations [42] and has been the focus of experiments in nonlinear optical systems [42, 43] and in fluid convection systems [44 46]. All these bifurcations are supercritical (i.e. second order) or very weakly subcritical. In a primary bifurcation the system goes from a homogeneous state to a patterned one (with broken symmetries). In a secondary bifurcation, the previous existing phase (pattern) could nonlinearly interact with the fluctuations. Thus, the dynamics of the critical modes show features that will be relevant similarly to primary bifurcations where the broken symmetry phase has more than one critical mode [44].

On the other hand, in subcritical bifurcations we have to distinguish two cases: weakly subcritical bifurcations where the causal mechanisms could hold for fast enough transitions, due to the existence of a slowing down of the relevant modes (although the relaxation times do not diverge) [44, 47] and others where the mechanisms are completely different [48].

In this paper, we show first experimental results on the quenching dynamics taking place in a convective network of oscillators driven by a quasi-1D heating through a secondary bifurcation. The basic pattern is a stationary multicellular pattern (ST) from which different heating ramps will send the system towards an oscillatory pattern throughout the presence of domains of traveling waves (TW) and mixed patterns of counter-oscillating waves over ST (ST/ALT). Furthermore, as we increase the quench intensity the system will cross a second bifurcation to the counter-oscillating pattern (ALT). This experiment has been studied in a previous experimental setup [49, 50] and an improved version can be found in 51, 52]. Therefore, this experiment allows us to study the quenching dynamics from a constrained degeneracy given by a nonhomogeneous basic pattern (ST). For this system we have already shown that the classical pattern formation can be understood from the point of view of Networks [1] as the phase synchronization between individual oscillators. In this sense, this work is very relevant because the results on the quenched dynamics in the critical region can be translated into the interaction and collective behavior of those oscillators. Notwithstanding the existence of very few numerical works [53] pointing towards this direction.

Although the classical Kibble-Zurek mechanism (its limitations and possible extensions) is still a matter of discussion [54, 55], we aim at finding new clues in experiments which could enlighten the Kibble-Zurek mechanisms from its foundations (i.e. causality and dynamical aspects of bifurcations). Specifically, we are going to focus for the first time on the fact that the studied bifurcation is secondary, and also we will consider its weak (but not very weak, though measurable) subcritical character.

\section{EXPERIMENTAL SETUP}

Our system consists on a rectangular cell $L_{x} \times L_{y}$ $\left(L_{x}=470 \mathrm{~mm}, L_{y}=60 \mathrm{~mm}\right)$ filled with a Silicone oil of viscosity $5 \mathrm{cSt}$. Convection is achieved by heating the fluid layer from below and along a central line (in the widest direction $\hat{x}$ ). The fluid layer lies over a plane plate with a heating rail underneath. Temperature on this underlying plate $\left(T_{b}\right)$ is selected at a heating bath $\left(T_{h}\right)$. The upper surface of the fluid layer is opened to the atmosphere and the depth of the fluid layer $d$ is measured with a micrometric screw. Lateral cooled walls $\left(T_{c}\right)$ and room temperature $\left(T_{a}\right)$ are kept at $20.0 \pm 0.1^{\circ} \mathrm{C}$. The control parameter for a fixed depth is the vertical temperature difference $\Delta T_{v}=T_{h}-T_{a}$ and the reduced control parameter is defined as $\varepsilon=\frac{\Delta T_{v}-\Delta T_{v c}}{\Delta T_{v}}$, where $\Delta T_{v c}=T_{h c}-T_{a}$ is the critical value at the threshold. A more detailed description of the present experimental setup can be found in Miranda and Burguete [51]. For the results reported here, we have been supplied with a different $5 c$ St Silicone oil whose only effect is to displace the convective thresholds.

During each quench, the temperature $T_{b}$ is recorded at the heating line and controlled at the entrance of the cell in order to check the heating bath response. Because the underlying plate is the most external structure from the inner core of the cell, we have obtained that $T_{b} \approx$ $T_{h}-9.0^{\circ} \mathrm{C}$.

The dynamics of the convective cells is recorded from the shadowgraphy images on the screen by placing an acquisition line (we use an image acquisition board connected to a CCD camera) next to the heating line. Each measurement consists on a spatial and temporal sampling image in grey levels which is a spatiotemporal diagram. Spatiotemporal diagrams show a central region of the cell of $156 \mathrm{~mm}$ long that is recorded during 900 seconds at a frequency of $1 \mathrm{~s}^{-1}$.

Due to the high thermal inertia of this system, before recording each spatiotemporal diagram it is necessary to achieve a permanent regime which takes at least three hours from an initial state where the whole cell is at room temperature.

\section{MEASUREMENT PROCESS}

On the stability diagram in Fig. 1, we set our control parameters at $\left(d=7.5 \mathrm{~mm}, \Delta T_{v}=16.0^{\circ} \mathrm{C}\right)$, close to the codimension- 2 point from below, in order to achieve a constant velocity at the static threshold of the secondary bifurcation $\varepsilon=0$ (see Fig. 2). Thus, the crossing rate is given by $\mu=\left.\frac{d T}{d t}\right|_{\varepsilon=0}$. This secondary bifurcation is weakly subcritical when it is crossed quasi-statically (with a subcriticality of $\epsilon \approx-0.02[51]$ ). Under these conditions, the system bifurcates from a multicellular pattern towards the oscillatory pattern ST/ALT+TW at the first subcritical threshold, or towards ALT at the second one depending on the quench power. We observe 


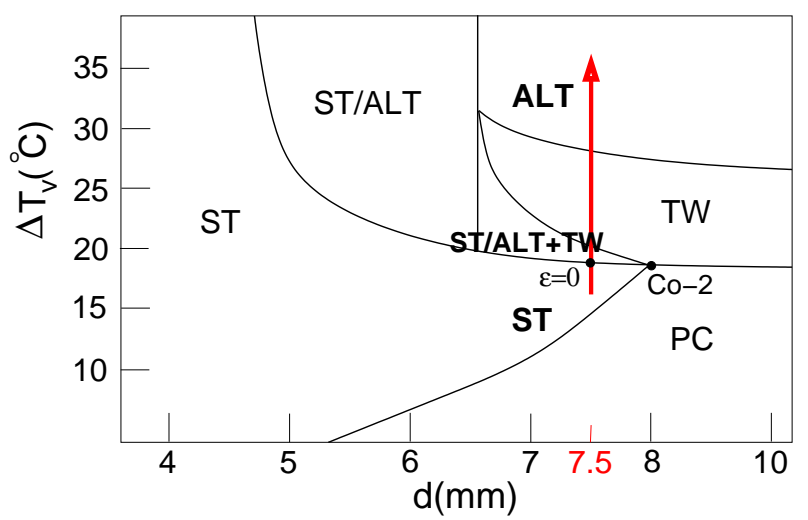

FIG. 1: Stability diagram. Solid lines bound regions with the same asymptotic dynamics. The upward arrow shows the range of quenches that has been explored at $d=7.5 \mathrm{~mm}$. Co2 stands for codimension-2 point. Stationary patterns are: PC (primary convection which, in our system, is the homogeneous state) and ST (multicellular pattern). Oscillatory patterns are: ALT (resonant triad), ST/ALT (mixed pattern of irregular clusters in ALT over ST), TW (traveling waves) and ST/ALT + TW (coexistence between the mixed pattern ST/ALT and TW).

from Fig. 1 1 that, beyond the codimension- 2 point $(d \geq 8$ $\mathrm{mm}$ ), the system bifurcates supercritically from a homogeneous state towards traveling waves.

The starting temperature selected at the heating bath is determined from the closeness to the static threshold $\varepsilon=0$ of the secondary bifurcation towards $\mathrm{ST} / \mathrm{ALT}+\mathrm{TW}$. This fact assures the linear behavior of temperature in the vicinity of $\varepsilon=0$. Thus, quenches start at the stationary regime ST which is characterized by a wavelength $\left(\lambda_{s}\right)$ that allows us to define an array of convective oscillators, initially at rest. In the course of quenches, these convective oscillators will become nonlocally coupled through the correlation length $\xi$. This correlation length is measured at the transition front.

Each heating ramp starts at $T_{h}=36.0^{\circ} \mathrm{C}$ (on the underlying plate the corresponding temperature is $T_{b}=$ $27.2 \pm 0.1^{\circ} \mathrm{C}$ ) and is characterized by the final temperature in the range from $T_{h}=42.0^{\circ} \mathrm{C}$ to $T_{h}=54.0^{\circ} \mathrm{C}$. In order to get a better accuracy, we have worked with steps of 1 or 2 degrees depending on the subcritical nature of the region to be studied.

The results reported here correspond to the average of four spatiotemporal diagrams for each heating ramp. For a given crossing rate $\mu$, we find out the corresponding transition front. We define this 1D-front as the spatial position where the new phase has been identified in time: $F_{\mu}(x, t)=0$. From now on, it will be expressed explicitly as $t=f_{\mu}(x)$, which is a univaluated function. Fronts are determined from the amplitude and phase diagrams because it is not possible to track the oscillators paths individually along the quenched dynamics. Trajectories described by each oscillator might become deformed along quenched regimes invading neighboring paths in order to

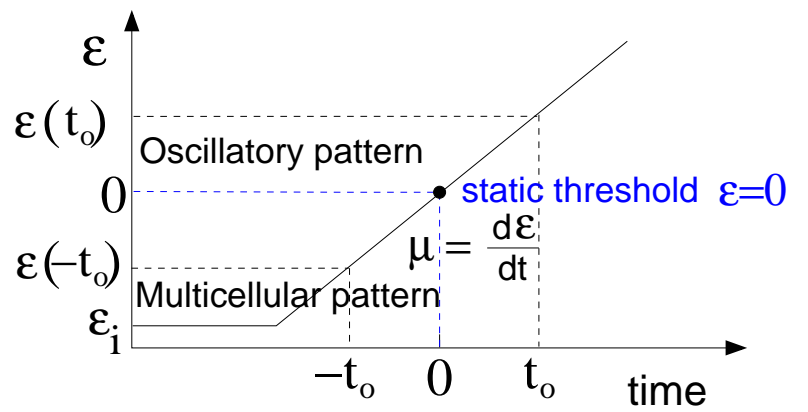

FIG. 2: Sketch of the control parameter ramp at the threshold of a secondary instability $\varepsilon=0\left(T_{b}=29.5^{\circ} \mathrm{C}\right)$. The slow relaxation dynamics takes place in the region $\left[-t_{o}, t_{o}\right]$ where the crossing rate is given by $\mu=\left.\frac{d \varepsilon}{d t}\right|_{\varepsilon=0}$. The initial value of the control parameter for each quench is $\varepsilon_{i}$ $\left(T_{b}=27.2^{\circ} \mathrm{C}\right)$ where the system exhibits the multicellular regime (ST). Above $\varepsilon=0$ the system bifurcates to an oscillatory pattern.

adjust to a new wavenumber and frequency. This fact has determined the necessity of analyzing the global phaseamplitude information of spatiotemporal diagrams for a constant illumination. Therefore, the front of active oscillators $t=f_{\mu}(x)$ is obtained by complex demodulation from the matrix of amplitudes $|A(x, t)| \geq \alpha\left|A_{\max }(x, t)\right|$, where $\left|A_{\max }(x, t)\right|$ is the maximum amplitude of the critical mode and $\alpha$ is a constant $(0<\alpha<1)$. Critical modes are selected with the following criteria: those with the highest amplitude in the Fourier spectrum, and those providing the dynamics at the front. Amplitude diagrams have been tested in the range $\alpha=[0.2-0.37]$ in order to assure that the shape of the front is conserved.

The self-correlation of the fronts is obtained as:

$$
\mathcal{C}_{\mu}(\zeta)=\int_{x}\left[f_{\mu}(x+\zeta)-\left\langle f_{\mu}(x)\right\rangle\right]\left[f_{\mu}(x)-\left\langle f_{\mu}(x)\right\rangle\right] d x .
$$

where $\zeta$ is the spatial lag and \langle\rangle defines the spatial average value. Hence, the characteristic correlation length $\xi$ is given by the spatial lag at $e^{-1}$ of the largest value of $\mathcal{C}_{\mu}$ :

$$
\xi=\min \left[\mathcal{C}_{\mu}^{-1}\left\{\frac{\mathcal{C}_{\mu}(0)}{e}\right\}\right] .
$$

The values of $\mathcal{C}_{\mu}(\zeta)$ have been computed for different parameters (amplitude thresholds and filter properties). From these values we have obtained a similar description of the dynamics, therefore we have introduced these deviations in the error bars computed for each spatiotemporal diagram.

Temperature measurements at the underlying plate are shown in Fig. [3] for a particular sequence of quenches. We implement a nonlinear curve fitting taking into account the heat transfer from the water circulation in the heating bath towards the underlying plate (see Fig. 3). For each temperature ramp, the crossing rate is obtained from the slope of each fitted curve at the static threshold: $\mu=$ $\left.\frac{d T_{b}}{d t}\right|_{\varepsilon=0}$. 


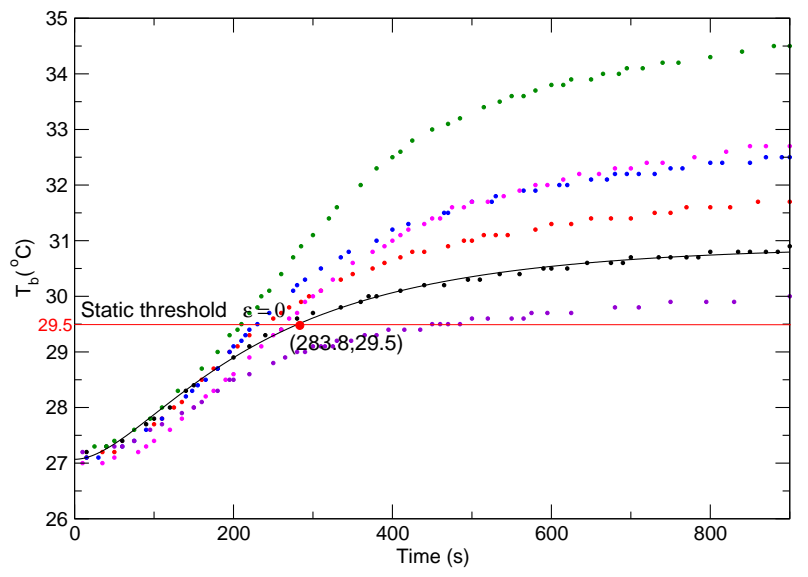

FIG. 3: Temperature measurements at the underlying plate of the cell belonging to a sequence of quenches. The static threshold of this secondary bifurcation $\varepsilon=0$ takes place at $T_{b}=29.5^{\circ} \mathrm{C}$. Each temperature profile has been fitted to the curve $T_{b}=a_{o}+a_{1} e^{-t / a_{2}}+a_{1}\left(1-e^{-t / a_{2}} e^{-t / a_{3}}\right)$. The solid line corresponds to the following parameter values: $a_{o}=30.91$, $a_{1}=-3.84, a_{2}=254.10, a_{3}=96.38$. The crossing rate in this particular case is $\mu=\frac{d T_{b}}{d t}\left(T_{b}=29.5^{\circ} \mathrm{C}\right)=0.0061^{\circ} \mathrm{C} / \mathrm{s}$.

\section{RESULTS AND DISCUSSION}

The initial pattern at the beginning of each quench is the stationary one (ST), with wavelength $\lambda_{s}=4.75 \pm 0.05$ $\mathrm{mm}$, which corresponds to an array of 95 latent oscillators, although we observe only the central region of the system (approximately 33 oscillators). Once the quenched regime bifurcates towards an oscillatory pattern, we obtain the corresponding front $t=f_{\mu}(x)$ by selecting the Fourier oscillatory modes with the minimum frequency in order to obtain the first unstable front from the multicellular pattern.

In Fig. 4, we show the wavenumbers and frequencies of the critical modes. From Fig. 4(a) we have measured an average wavelength of $8.76 \pm 0.01 \mathrm{~mm}$ for $\mu \leq 0.0095^{\circ} \mathrm{C} / \mathrm{s}$, and of $9.25 \pm 0.02 \mathrm{~mm}$ for $\mu>0.0095^{\circ} \mathrm{C} / \mathrm{s}$. The gap between these values might be inherited from two different and consecutive subcritical thresholds in the permanent regimes [51]. This observation will be checked with the corresponding spatiotemporal diagrams and fronts further on.

From Fig. 4(b) we obtain an average period of $23.60 \pm$ $0.01 \mathrm{~s}$ which remains practically constant at any crossing rate. A detailed analysis of the spatiotemporal diagrams shows that the frequency evolves slowly along the heating ramp as it is expected from previous results [51]. This fact is related to the slow response of the system towards the transition point where the modes with the lowest frequencies are the very first to guide this quenched bifurcation.

In Figs. [5]6] we show some spatiotemporal diagrams that represent the quenching dynamics along two different and contiguous subcritical thresholds. Also, the cor-

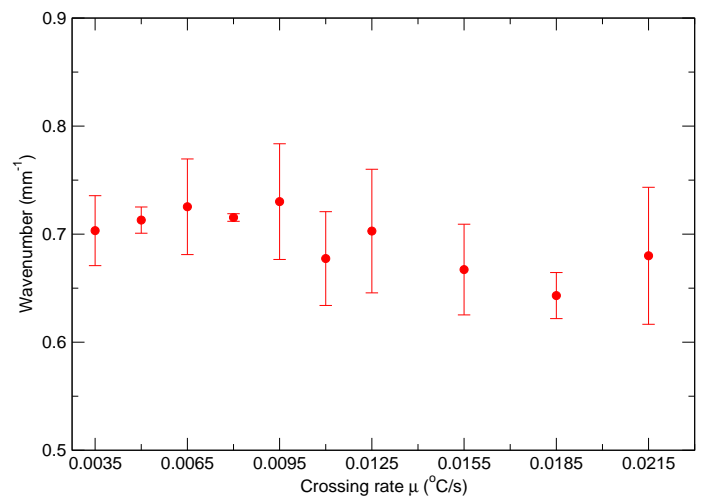

(a)

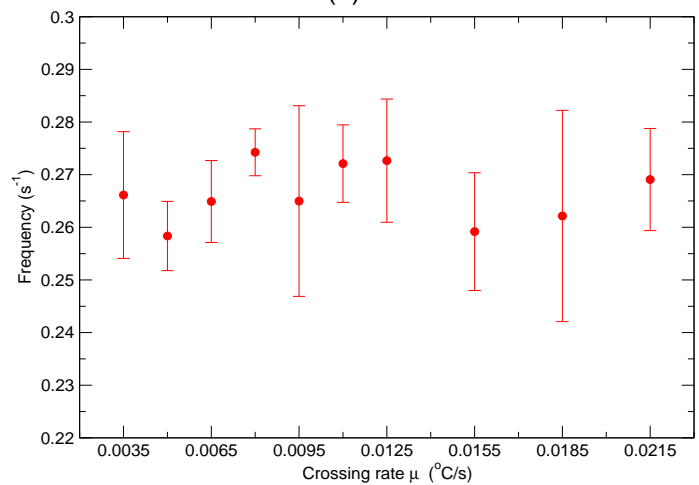

(b)

FIG. 4: Wavenumbers (a) and frequencies (b) of the critical modes that define the front at a given quench. Error bars are given by the dispersion of values given by the left and right oscillatory modes for different sequences.

responding fronts $t=f_{\mu}(x)$ have been overlapped. We briefly summarize the main features of these transitions when they are crossed quasi-statically (for a more detailed study of the quasi-static transitions see Miranda and Burguete [51]):

(I) In the first subcritical bifurcation, left and right traveling waves (with $\lambda_{T W \pm} \approx 1.5 \lambda_{s}$ ) are bounded in domains which coexist with the mixed pattern ST/ALT (with $\lambda_{A L T} \approx 2 \lambda_{s}$ ).

(II) In the second subcritical bifurcation, the ALT pattern is expected to coexist with the TW pattern until, for increasing values of the control parameter, the only remaining pattern is the ALT one.

In Fig. 15 we show the transition front for different crossing rates ranging from $\mu=0.0035^{\circ} \mathrm{C} / \mathrm{s}$, with a spatio-temporal coherent front, until the crossing rate reaches $\mu=0.0125^{\circ} \mathrm{C} / \mathrm{s}$, with a more homogeneous front. We observe that the front becomes fragmented in between as the quench power is increased. Thus, we may infer that, in the range shown in this figure, the front exhibits different degrees of coherence depending on the strength of coupling between oscillators.

In Fig. 6. the bifurcation scenario is more robust because from the previous quasi-homogeneous front (e.g. 


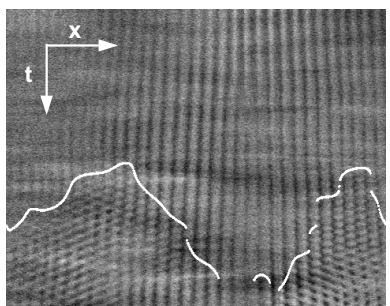

(a)

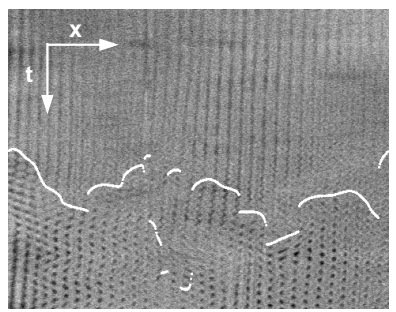

(c)

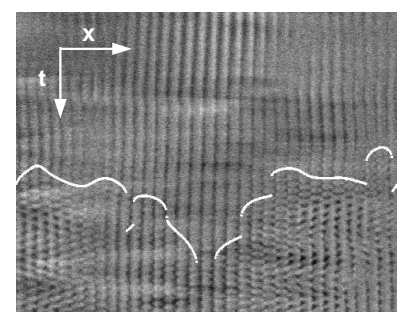

(b)

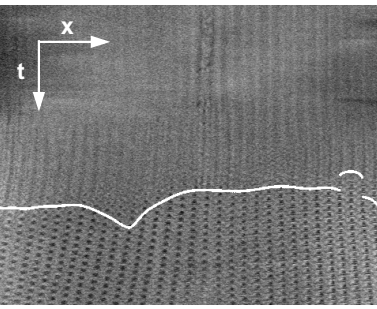

(d)
FIG. 5: Spatiotemporal diagrams corresponding to quenched dynamics: (a) $\mu=0.0035^{\circ} \mathrm{C} / \mathrm{s}$, (b) $\mu=0.0065^{\circ} \mathrm{C} / \mathrm{s}$, (c) $\mu=$ $0.0095^{\circ} \mathrm{C} / \mathrm{s}$, (d) $\mu=0.0125^{\circ} \mathrm{C} / \mathrm{s}$. White lines correspond to the fronts defined by left oscillatory modes.

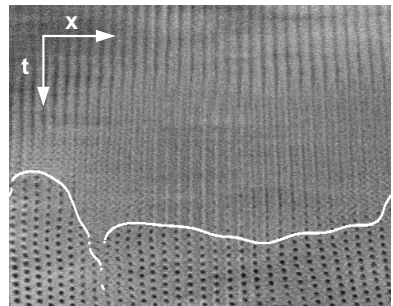

(a)

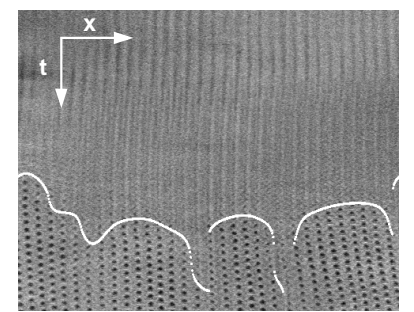

(b)
FIG. 6: Spatiotemporal diagrams corresponding to quenched dynamics: (a) $\mu=0.0155^{\circ} \mathrm{C} / \mathrm{s}$, (b) $\mu=0.0215^{\circ} \mathrm{C} / \mathrm{s}$. White lines correspond to the fronts defined by left oscillatory modes.

see Fig. $5(\mathrm{~d})$ ) topological defects arise at the front. These defects have survived the quenched transition and remain pinned to the transition front.

From these patterns we can distinguish two different behaviors:

(i) For $\mu \leq 0.0095^{\circ} \mathrm{C} / \mathrm{s}$, the quenching dynamics after the onset of the bifurcation shows the presence of certain domains in the TW and ST/ALT patterns which are remnants of the subcritical behavior of the quasi-static transition. We should stress that the front describes the time at which each oscillator bifurcates disregarding the fluctuating behavior of the oscillators that belong to the mixed pattern ST/ALT (because they are susceptible of returning to the stationary state).

(ii) For $\mu>0.0095^{\circ} \mathrm{C} / \mathrm{s}$, the quenching dynamics sends the system through the following subcritical bifurcation. Nevertheless, the new pattern has entirely bifurcated to the ALT pattern. Therefore, for fast

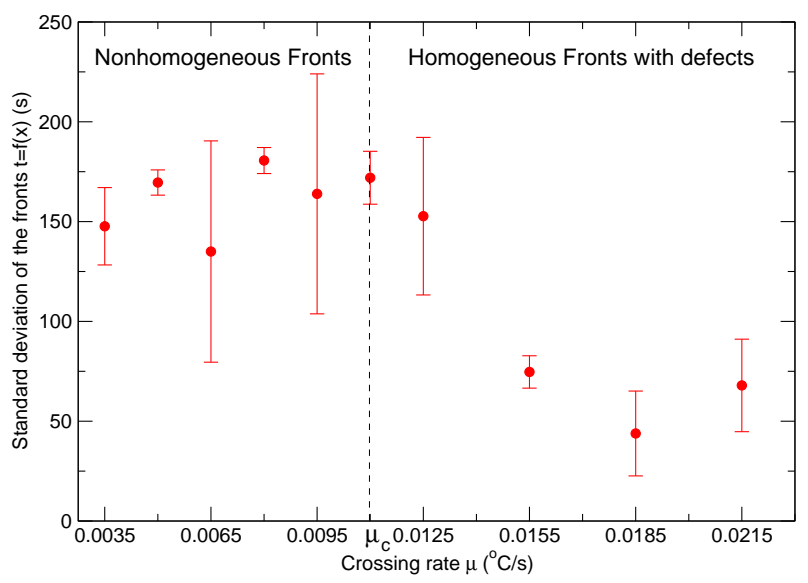

FIG. 7: Standard deviation of the fronts versus the crossing rate $\mu$. The dashed line indicates the critical value $\mu_{c}$.

quenches, during the relaxation time that takes the system to reach the onset of this bifurcation, the most unstable mode is the one that represents the ALT pattern (which doubles the wavelength of the multicellular pattern).

In Fig. 7, we measure for different sequences the degree of inhomogeneity of the front given by the standard deviation of the front $t=f_{\mu}(x)$. We have taken into account fronts defined from both counter-oscillating modes. These modes define similar fronts except for the very slow values of $\mu$ because of the presence of TW domains that are attached to the front. We should emphasize that results from Fig. 7 match the dynamical description of the fronts given above: for slow crossing rates nonhomogeneous fronts appear, meanwhile as the crossing rate is increased the fronts become more homogeneous and robust, this fact is related to the stronger coupling between oscillators [1].

In Fig. 8, we show the correlation length of the fronts $\xi$ which is obtained from Eq. (2). The maximum value of $\xi$ corresponds to a quasi-homogeneous front (see Fig. 5 (d)) at a critical value of the crossing rate $\mu_{c}$. For slow quenches $\left(\mu<\mu_{c}\right)$, there is a minimum correlation length that belongs to the kind of fragmented fronts shown in Fig. 5(b,c). In these fronts, neighboring oscillators are beginning to show a stronger coupling [1]. On the other hand, according to the Kibble-Zurek mechanism in phase transitions our experimental results for fast quenches $\left(\mu>\mu_{c}\right)$ follow a power law despite the critical exponent is far to follow previous experimental results obtained in other systems.

In Fig. 9, the minimum time that is needed to bifurcate from the static threshold is a measure of the relaxation time of the convective array of oscillators. We observe that for the slowest quenches, when the oscillators are weakly coupled, the relaxation time is the shortest. But as the power law behavior is reached (for $\mu>\mu_{c}$ ) the relaxation time becomes much longer as if the quenched dynamics from the basic pattern ST to the ALT pattern 


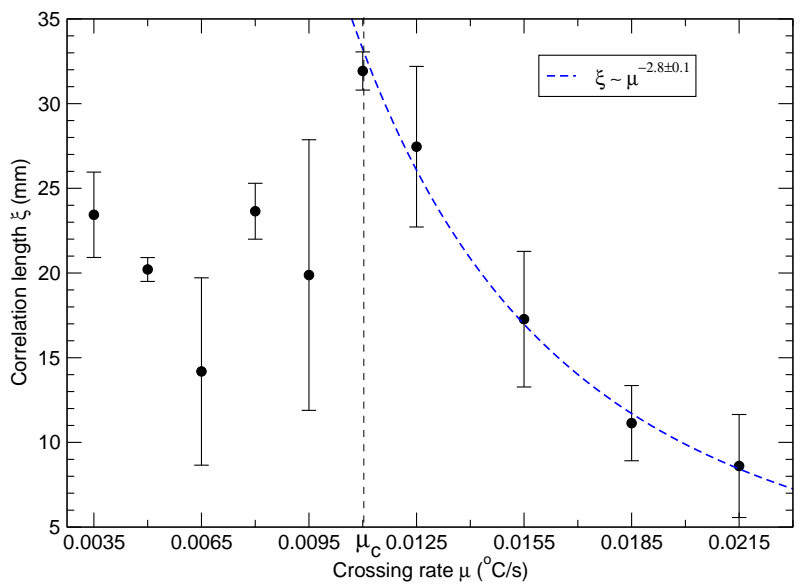

FIG. 8: Self-correlation of the fronts $\xi$ versus the crossing rate $\mu$. The vertical dashed line indicates the critical value $\mu_{c}$.

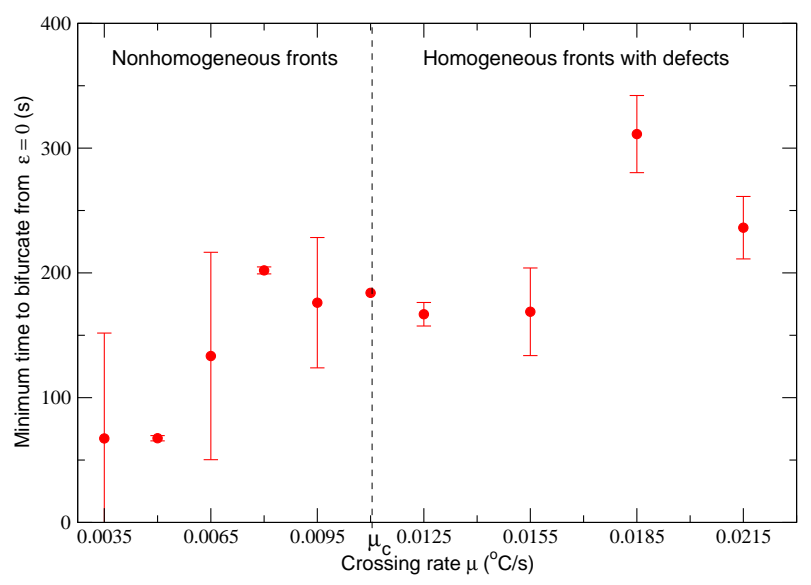

FIG. 9: Minimum time to bifurcate from the static threshold $\varepsilon=0$ versus the crossing rate $\mu$. The dashed line indicates the critical value $\mu_{c}$.

had corresponded to that of a supercritical bifurcation (see Fig. 6).

\section{FURTHER REMARKS AND CONCLUSIONS}

In this paper we report experimental evidence of the slow response dynamics arising from quenched transitions in a quasi-1D convective system. The quenched dynamics goes through a secondary and weakly subcritical bifurcation for a depth of the fluid layer of $d=7.5 \mathrm{~mm}$. Complementary results concerning quenches through a stronger subcritical bifurcation are going to be reported elsewhere [56].

Specifically, these results correspond to the kind of ramped symmetry breaking bifurcations where an initially quasi-degenerated state (ST) undergoes a phase transition where it looses some symmetries towards the oscillatory patterns (ST/ALT+TW or ALT). This dynamics can be characterized from the shape of the fronts

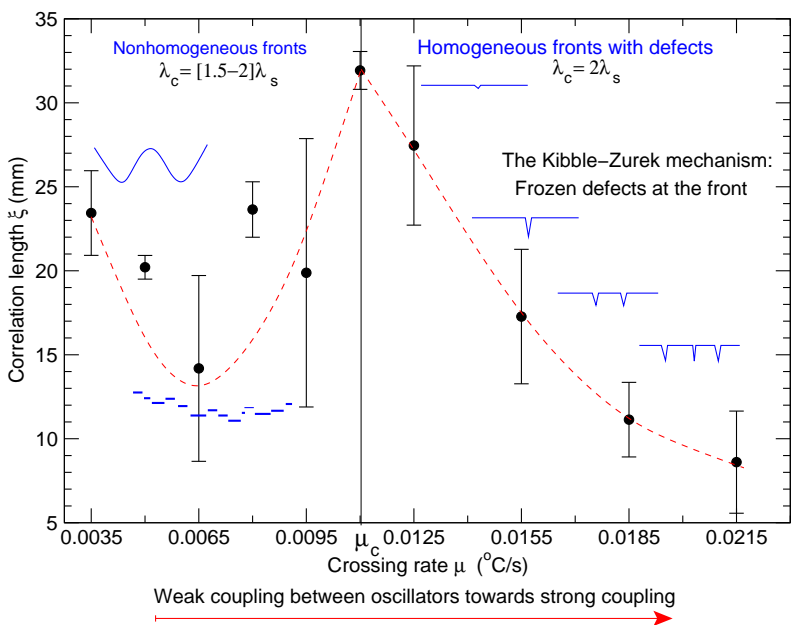

FIG. 10: Sketch of the quenching mechanism. Blue lines represent fronts while dashed lines are a guide to the eye. The vertical solid line indicates the critical value $\mu_{c}$.

where defects become trapped above a critical value of the crossing rate $\mu_{c}$. Fronts and topological defects are the kind of localized coherent structures that have survived a sudden transition in the laboratory, and represent an interesting analogy to the cosmological objects after the Big Bang [57].

According to the Kibble-Zurek mechanism, the distance between defects might revel the order of the correlation length scale which diverges from the correlation length $\xi$ measured from the fronts. If the positions of defects along the array are uncorrelated, one may think that the distribution of defects along the fronts does not respond to the healing length and perhaps to a nonlinear mechanism regarding the distant position of the last subcritical instability from the primary bifurcation. For $\mu>\mu_{c}$, despite the analysis could have been done taking as reference the next bifurcation, we choose not to; because in fact the pattern for $\varepsilon<0$ (ST) remains unchanged until the studied front is revealed.

The fronts are expected to show the degree of interaction between the convective oscillators along the network. In Fig. 10, we summarize the most relevant aspects of this paper. We should outline that the results on the correlation length are a measure of the degree of interaction between oscillators. On the other hand, fluctuations of the front that have not been absorbed by the front during the slow relaxation time, do represent an order parameter of the quenched dynamics. In consequence, the shape of the front is a trace of the slow relaxation dynamics at the critical point of a symmetry breaking bifurcation.

\section{Acknowledgments}

This work has been partly supported by the Spanish Contract No. FIS2008-01126 and by the Gobierno of Navarra (Departmento de Educación). 
[1] M. A. Miranda and J. Burguete, Int. J. Bifurcation and Chaos 20(3), 835 (2010).

[2] D. Mertens and R. Weaver, Complexity (2010), ISSN 1099-0526.

[3] H. Mancini and G. Vidal, To appear in EPJ D Topical issue in Instabilities and Nonequilibrium structures (accepted) (2010).

[4] A. Arenas, A. Díaz-Guilera, J. Kurths, Y. Moreno, and C. Zhou, Phys. Rep. 469, 96 (2008).

[5] G. V. Osipov, J. Kurths, and C. Zhou, Synchronization in oscillatory networks (Springer-Verlag, Berlin, 2007).

[6] D. M. Abrams and S. H. Strogatz, Int. J. Bifurcation and Chaos 16(1), 21 (2006).

[7] C. Zhou and J. Kurths, Phys. Rev. Lett. 96, 164102 (2006).

[8] S. Boccaletti, Y. Moreno, M. Chavez, and D.-U. Hwang, Phys. Rep. 424, 175 (2006).

[9] S. Shima and Y. Kuramoto, Phys. Rev. E 69, 036213 (2004).

[10] Y. Kuramoto, Chemical oscillations, waves and turbulence (Dover, 2003).

[11] G. S. Guralnik, C. R. Hagen, and T. W. B. Kibble, Phys. Rev. Lett. 13, 585 (1964).

[12] T. W. B. Kibble, J. Phys. A9, 1387 (1976).

[13] T. W. B. Kibble, Phys. Rep. 67, 183 (1980).

[14] H. Muruyama and J. Shu, Phys. Lett. B 686, 162 (2010).

[15] W. H. Zurek, Nature 317, 505 (1985).

[16] W. H. Zurek, Phys. Rep. 276, 177 (1996).

[17] A. Rajantie, Int. J. Mod. Phys. A 17, 1 (2002).

[18] A. Rajantie, Contemp. Phys. 44, 485 (2003).

[19] J. Dziarmaga, Advances in Physics 59: 6, 10631189 (2010).

[20] I. Chuang, R. Dürrer, N. Turok, and B. Yurke, Science 251, 1336 (1991).

[21] M. J. Bowick, L. Chandar, E. A. Schiff, and A. M. Srivastava, Science 263, 943 (1994).

[22] S. Digal, R. Ray, and A. M. Srivastava, Phys. Rev. Lett. 83, 5030 (1999).

[23] P. C. Hendry, N. S. Lawson, R. A. M. Lee, P. V. E. McClintock, and C. D. H. Williams, Nature 368, 315 (1994).

[24] C. Bäuerle, Y. M. Bunkov, S. N. Fischer, H. Godfron, and G. R. Pickett, Nature 382, 332 (1996).

[25] V. M. Ruutu, V. B. Eltsov, A. J. Gill, T. W. B. Kibble, M. Krusius, Y. G. Makhlin, B. Placais, G. E. Volovik, and W. Xu, Nature 382, 334 (1996).

[26] V. M. Ruutu, V. B. Eltsov, M. Krusius, Y. G. Makhlin, B. Placais, and G. E. Volovik, Phys. Rev. Lett. 80, 1465 (1998).

[27] V. B. Eltsov, T. W. B. Kibble, M. Krusius, V. M. H. Ruutu, and G. E. Volovik, Phys. Rev. Lett. 85, 4739 (2000).

[28] Y. Bunkov, Physica B 329, 70 (2003).

[29] V. B. Eltsov, M. Krusius, and G. Volovik, condmat/9809125 v4 (2004).
[30] V. Eltsov, R. Hänninen, and M. Krusius, Physica C 470, 803 (2010).

[31] R. Carmi and E. Polturak, Phys. Rev. B 60, 7595 (1999).

[32] R. Monaco, R. J. Rivers, and E. Kavoussanaki, J. Low Temp. Phys. 124, 85 (2001).

[33] R. Monaco, J. Mygind, and R. J. Rivers, Phys. Rev. Lett. 89, 080603 (2002).

[34] R. Monaco, J. Mygind, and R. J. Rivers, Phys. Rev. B 67, 104506 (2003).

[35] R. Monaco, U. L. Olsen, J. Mygind, R. J. Rivers, and V. P. Koshelets, Phys. Rev. Lett 96, 180604 (2006).

[36] R. Monaco, M. Aaroe, J. Mygind, R. J. Rivers, and V. P. Koshelets, Phys. Rev. B 77, 054509 (2008).

[37] R. Monaco, J. Mygind, R. J. Rivers, and V. P. Koshelets, Phys. Rev. B 80, 180501 (2009).

[38] A. Maniv, E. Polturak, and G. Koren, Phys. Rev. Lett. 91, 197001 (2003).

[39] C. Weiler, T. Neely, D. Scherer, A. Bradley, M. Davis, and B. Anderson, Nature 455, 948 (2008).

[40] L. Sadler, J. Higbie, S. Leslie, M. Vengalattore, and D. Stamper-Kurn, Nature 443, 312 (2006).

[41] R. Yusupov, T. Mertelj, V. Kabanov, S. Brasovskii, P. Kusar, J.-H. Chu, I. Fisher, and D. Mihailovic, Nature Phys. (2010).

[42] S. Casado, W. González-Viñas, S. Boccaletti, P. L. Ramazza, and H. Mancini, Eur. Phys. J. Special Topics 146, 87 (2007).

[43] S. Ducci, P. L. Ramazza, W. González-Viñas, and F. T. Arecchi, Phys. Rev. Lett. 83, 5210 (1999).

[44] S. Casado, W. González-Viñas, H. Mancini, and S. Boccaletti, Phys. Rev. E 63, 057301 (2001).

[45] W. González-Viñas, S. Casado, J. Burguete, H. Mancini, and S. Boccaletti, Int. J. Bif. Chaos 11, 2887 (2001).

[46] S. Casado, Ph.D. thesis, University of Navarra (2002).

[47] R. Schützhold, J. Low Temp. Phys. 153, 228 (2008).

[48] T. Vachaspati, Kinks and Domain Walls (Cambridge University Press, 2006).

[49] J. Burguete, H. L. Mancini, and C. Pérez-García, Europhys. Lett. 23, 401 (1993).

[50] J. Burguete, D. Maza, and H. L. Mancini, Physica D 174, 56 (2003).

[51] M. A. Miranda and J. Burguete, Phys. Rev. E 78, 046305 (2008).

[52] M. A. Miranda and J. Burguete, Phys. Rev. E 79, 046201 (2009).

[53] M. Ciszak, A. Montina, and F. Arecchi, Cogn. Process 10, 33 (2009).

[54] E. Moro and G. Lythe, Phys. Rev. E 59, 1303 (1999).

[55] G. Biroli, L. Cugliandolo, and A. Sicilia, Phys. Rev. E 81, 050101(R) (2010).

[56] M. A. Miranda, J. Burguete, W. González-Viñas, and H. Mancini, p. (in preparation) (2011).

[57] T. W. B. Kibble, Physics Today pp. September 47-52 (2007). 